\title{
Estudo da prevalência de enteroparasitoses em pacientes de um laboratório privado do município de Santarém
}

\author{
Study of the prevalence of enteroparasitoses in patients in a private laboratory in the municipality \\ of Santarém
}

\author{
Daniele Catrine de Sousa Cunha Lima* ${ }^{\bullet}$, Patrícia Spinola da Rocha ${ }^{\circ}$
}

Centro Universitário da Amazônia, Santarém, Pará, Brasil. *Autor para correspondência. E-mail: danielecunhaa_@outlook.com.

\begin{abstract}
Resumo: Introdução: As enteroparasitoses ainda são marcadas como um problema de saúde pública mundial, principalmente em países subdesenvolvidos ou em desenvolvimento, o que ocorre devido a fatores relacionados a questões socioeconômicas, ambientais e culturais. Objetivo: A pesquisa objetivou fornecer o perfil epidemiológico de enteroparasitoses em pacientes atendidos no laboratório de análises clínicas privado, relatando a prevalência e frequência das enteroparasitoses em crianças, adolescentes e adultos residentes na cidade de Santarém-PA. Material e métodos: Estudo de caráter transversal descritivo analítico, realizado através da análise de laudos parasitológicos referentes a pacientes atendidos em um laboratório privado do baixo amazonas. Resultados e discussão: Mediante os resultados obtidos na pesquisa, foi notório que a prevalência de enteroparasitoses mostrou-se maior em crianças na faixa etária de 1-10 anos (54\%), sendo a maioria dos infectados o sexo feminino, bem como na idade infantil e adulta. Considerações finais: Através dos resultados obtidos é evidente que se faz necessária a melhoria na condição sanitária básica e na educação em saúde para conscientização da população sobre os riscos e prevenção de parasitoses, proporcionando assim, qualidade de vida à comunidade.
\end{abstract}

Palavras-chave: enteroparasitoses, Endolimax nana, protozoários, helmintos, pesquisa.

\begin{abstract}
Introduction: Enteroparasitosis are still marked as a global public health problem, which occurs due to factors related to socioeconomic, environmental and cultural issues, which occurs due to factors related to socioeconomic, environmental and cultural issues. Objective: The research aimed to provide the epidemiological profile of enteroparasitosis in patients treated in the private clinical analysis laboratory, reporting the prevalence and frequency of enteroparasitosis in children, adolescents and adults living in the city of Santarem-PA. Material and methods: This is a cross-sectional descriptive analytical study, carried out through the analysis of parasitological reports referring to patients treated in a private laboratory in the lower amazon region. Results and discussion: Based on the results obtained in the research, it was notorious that the prevalence of intestinal parasitosis was higher in children aged 1-10 years (54\%), with the majority of those infected being female, as well as in childhood and adulthood. Final considerations: Through the results obtained, it is evident that it is necessary to improve the basic health condition and health education to raise awareness of the population about the risks and prevention of parasites, thus providing quality of life to the community.
\end{abstract}

Keywords: enteroparasitosis, Endolimax nana, protozoa, helmi, search.

\section{Introdução}

No decorrer da evolução humana, muitas doenças que estiveram presentes em diferentes momentos históricos da sociedade disseminaram diversas enfermidades, de gigantesca relevância médica, apontando um significativo destaque clínico às parasitoses intestinais (Silva, 2017). As enteroparasitoses são originadas pela existência de helmintos e/ou protozoários no organismo de seres vivos, apresentam parte de seu ciclo evolutivo no sistema digestivo humano, o que, por sua vez, dá origem a quadros clínicos com efeitos danosos aos indivíduos (Silva et al., 2018). Os principais parasitas que acometem adultos e crianças são identificados como protozoários - Giárdia duodenale e Entamoeba histolytica/dispar e o comensal Entamoeba coli - e helmintos - Ascaris lumbricoides, Ancylostoma duodenale, Trichuris trichura (Bacelar et al., 2018). De acordo com dados da Escola Nacional de Saúde Pública da Fundação Oswaldo Cruz (Fiocruz), baseada em informações fornecidas pela Organização Mundial de Saúde (OMS), as parasitoses intestinais mostram-se com destaque na elevada taxa de morbidade. Exibindo em torno de 3,5 bilhões de pessoas infectadas por helmintos e/ou protozoários, sendo que por volta de 450 milhões deste infectados correspondem a crianças (Silva, 2017; Frei et al., 2008). 
As infecções parasitárias instalam-se geralmente através da via fecal-oral, tanto em crianças como em adultos, por meio da ingestão de água e/ou alimentos contaminados (principalmente os consumidos in natura). Além dessas formas de contágio, hábitos relacionados à própria infância tonam-se formas de transmissão muito comuns, como andar descalço em contato direto com o solo e o ato de levar à boca objetos (brinquedos) que possam estar contaminados (Ferreira et al., 2012). Já nos adultos, mais comumente, a contaminação ocorre pela ingestão de alimentos consumidos crus (vegetais).

Ainda que as parasitoses possam estar presentes em qualquer indivíduo, seja qual for a fase de sua vida, ao se estabelecer uma relação de comparação entre as faixas etárias, as crianças, sobretudo aquelas que estão em fase pré-escolar e escolar, ainda são o grupo de maior vulnerabilidade se comparados aos adolescentes e adultos (Ferreira et al., 2012). A predisposição em crianças é resultante de fatores imunológicos e de fatores característicos da própria idade, enquanto que em adolescentes e adultos a melhoria nos costumes relacionados à higiene e ao amadurecimento imunológico levam à explicação do motivo pela queda na contaminação (Camello et al., 2016; Nascimento, 2016; Dias et al., 2013).

Apesar de apresentar uma grande incidência, as enteroparasitoses ainda são avaliadas como um problema de saúde pública mundial, principalmente em países subdesenvolvidos ou em desenvolvimento, que apresentam fatores passíveis de tornar a infecção parasitaria suscetível. A prevalência dessas parasitoses manifesta diversidade em todo o mundo, conforme as condições socioeconômicas (Silva et al., 2018; Ferreira et al., 2012). No Brasil, por sua ampla distribuição geográfica, esse cenário ainda se faz presente em boa parte do território, principalmente nas regiões norte e nordeste, em razão da precariedade sanitária da população, sendo outros fatores motivados por questões ambientais e culturais, além dos fatores socioeconômicos, capazes de atingir áreas rurais e urbanas (Deniur et al., 2018; Barbosa \& Vieira, 2013).

As pesquisas realizadas evidenciaram que, apesar da ampla quantidade de pessoas parasitadas na região norte, os registros por meio de artigos referentes ao assunto são escassos, em especial no estado do Pará. Tal fato decorre da negligência no apontamento de casos informados, o que se torna um empecilho à realização de trabalhos de pesquisa, engendrando resultados não mais que pontuais, sutis e pouco abrangentes.

Em função da insuficiência de relatos direcionada às parasitoses em indivíduos habitantes da região do Pará, o trabalho em questão visa mediar informações, com o escopo de auxiliar futuros pesquisadores ao fornecer-lhes o perfil epidemiológico de enteroparasitoses em pacientes atendidos no laboratório de análises clínicas privado, com a certeza de contribuir de maneira positiva para o conhecimento epidemiológico, prevalência e frequência das enteroparasitoses em crianças, adolescentes e adultos residentes na cidade de Santarém-PA.

\section{Material e métodos}

Expondo caráter transversal descritivo analítico, o estudo apresenta como base a avaliação de laudos parasitológicos que já foram analisados pelo laboratório de apoio, liberados mediante a assinatura do Termo de Compromisso da Utilização de Dados (TCUD).

Os dados oferecidos à apreciação foram retirados dos registros dos pacientes que realizaram os exames no laboratório de análises clínicas privado, localizado no município de Santarém-PA, com uma área territorial de $17.898,389 \mathrm{~km}^{2}$, sendo sua população estimada no ano de 2021 de 308.339 habitantes, de acordo com dados do Instituto Brasileiro de Geografia e Estatística (IBGE) (IBGE, 2021).

Para o desenvolvimento do trabalho, foram coletados e investigados laudos de janeiro de 2020 a janeiro de 2021, considerando-se como critérios de inclusão: I) idade, II) sexo (masculino e feminino) e III) positividade parasitológica. Os dados recolhidos foram submetidos à planilha eletrônica do Microsoft Office Excel ${ }^{\circledR}$, versão 2019 , na qual foram analisados de forma descritiva e expressos em percentual.

No laboratório onde foi realizada a pesquisa, utilizam-se os métodos direto e de Hoffman para análise das amostras fecais, sendo inicialmente utilizado o método direto para reconhecimento dos parasitas e o método de Hoffman para confirmação. No primeiro, as fezes são examinadas mediante esfregaço em lâmina com uma gota de lugol para diluição, ideal para detecção de cistos de protozoários, trofozoítos e larvas de helmintos. Já o método de Hoffman é utilizado para pesquisa de ovos pesados e larvas de outros helmintos.

\section{Resultados e discussão}

Tratando-se de um país que ainda passa pelo processo de desenvolvimento e possuindo variações climáticas, sendo os mais predominantes climas tropical, equatorial e subtropical, o Brasil proporciona condições favoráveis para que os parasitas sobrevivam e se disseminem por todo o território (Sousa et al., 
2018). Em consequência ao vasto índice de parasitose por todo o território nacional, é possível estabelecer uma correlação entre a quantidade de indivíduos parasitados e a questão sanitária, de higiene e desenvolvimento socioeconômico (Damasceno \& Costa, 2016).

No estudo foram coletados 272 laudos parasitológicos, já analisados e liberados pelo laboratório de apoio, referentes ao período de $01 / 20$ a $01 / 21$. Expondo a pesquisa um caráter descritivo referente as idades de 1 aos 25 anos, de ambos os sexos, determinou-se as idades de 1-10 anos (crianças) e de 11-25 anos (adolescentes/adultos). Apresentando os valores de 146 crianças, 87 mulheres e 39 homens, tendo os resultados percentuais demonstrados no gráfico 1.

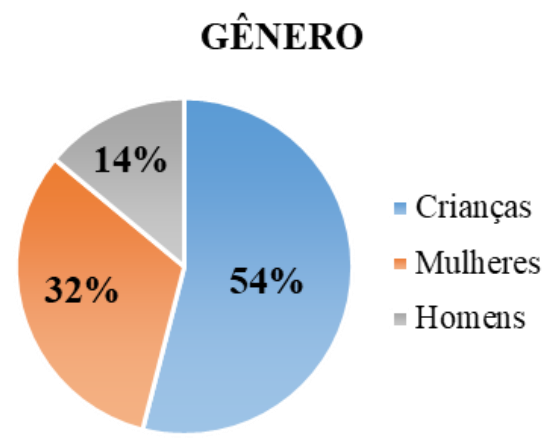

Gráfico 1. Percentual de parasitos quanto ao gênero dos hospedeiros.

Observou-se que a partir dos dados coletados através dos EPF, houve maior prevalência de enteroparasitoses em crianças, totalizando 54\% (145/272), o que corrobora com a maior parte das pesquisas já realizadas. Segundo Araujo Filho et al. (2011), as crianças compreendem ao grupo mais susceptível a infecções parasitarias, o que se deve a aspectos como idade, hábitos de higienização, estado nutricional, resposta imune e associação com outras alterações patológicas (Frei et al., 2008; Deniur et al., 2018), favorecendo assim as condições necessárias para que o ciclo evolutivo dos parasitos aconteça.

Já entre adolescentes e adultos, a incidência de enteroparasitoses mostrou-se relativamente menor, com um percentual de $46 \%$, o que se apresentou coerente com o que é descrito por Vasconcelos et al. (2011) sofrendo os quadros de parasitismo uma significativa redução com a maturidade, que ocorre pela melhoria na higiene pessoal e, principalmente, pelo amadurecimento imunológico, o que torna os adolescentes e adultos assintomáticos e transmissores ativos de parasitoses (Vasconcelos et al., 2011).

Considerando a distribuição parasitologia segundo a faixa etária do hospedeiro, assim como demonstrado no gráfico 2 , a frequência de casos apresentou maior destaque em crianças de 1 aos 10 anos - com maior frequência em crianças de 1 ano $(23,28 \%)$ e 5 anos (17,80\%) -, em comparação as demais faixas etárias, que exibiram um total de $46,32 \%$. Levando em consideração as determinantes do trabalho, foi notório que o gênero feminino, assim como na idade infantil quanto na idade adulta, mostrou-se maior, o que pode ser explicado pelo cuidado maior que o gênero feminino possui, ao estarem atentas a sintomas fora do padrão e frequentarem mais os médicos.

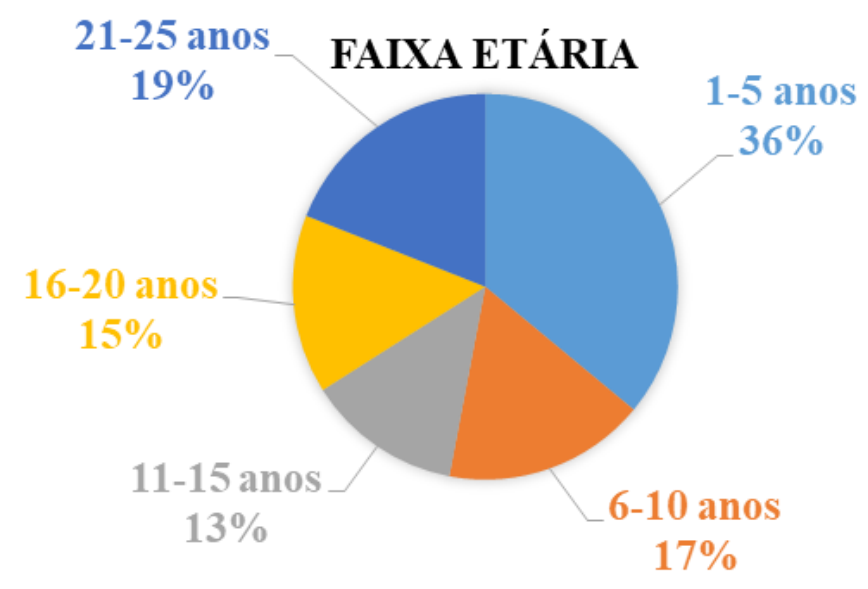

Gráfico 2. Percentual de parasitos quanto a idade dos hospedeiros. 
No gráfico 3, a predominância dos parasitos intestinais nos pacientes estudados pôde ser observada com a presença de protozoários e helmintos. Tendo uma maior frequência de protozooses, com casos isolados de helmintíases, a maioria sendo acompanhados pela presença de protozoários. Especificando 263 pacientes parasitados por protozoários e 9 por helmintos.

\section{TIPO DE PARASITO}

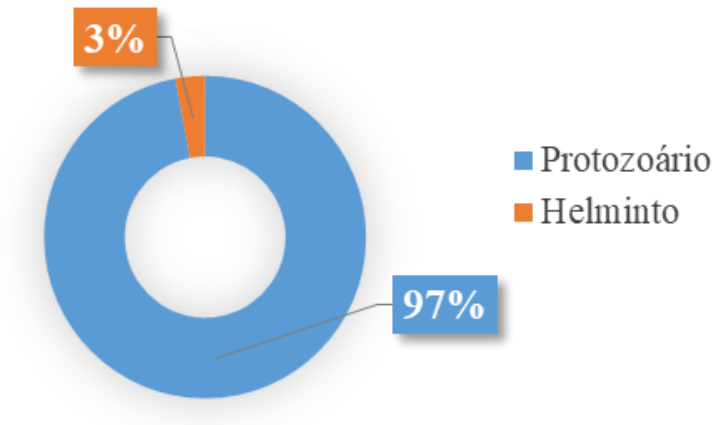

Gráfico 3. Percentual de parasitos quanto ao tipo.

Santos (2020) apresentam resultados aproximados à pesquisa realizada, com valores de 90,3\% para protozoários e de 16,5\% para helmintos (Santos, 2020). Dias et al. (2013) mostra em suas pesquisas protozoários 97,5\% e helmintos 8,3\%. (8) Resultados que se diferem drasticamente de Marques et al. (2021) onde os resultados de helmintos $(77,63 \%)$ mostram-se maior que os valores referentes a protozoários $(71,92 \%)$ (Marques et al., 2021). Correspondendo os respectivos artigos a localidades que fazem parte da região norte, é possível perceber que há um pequeno conflito de dados, o que pode ser explicado, além da questão sanitária, pela questão climática, Oriximiná e Macapá apresentando clima equatorial e Breves clima equatorial e topical (Curado, 2020).

A tabela 1 enumera a prevalência específica dos parasitos nos casos estudados, sendo esses classificados como protozoários e helmintos. Foram encontradas 10 espécies de parasitos, sendo 6 protozoários e 4 helmintos, destacando-se como espécies mais comuns: Cisto de Endolimax nana (179), Cisto de Blastocystis hominis (79) e Cisto de Entamoeba coli (78).

Tabela 1. Percentual de espécies parasitarias encontradas.

\begin{tabular}{ccc}
\hline \multicolumn{3}{c}{ Espécies de parasitos encontrados } \\
\hline Parasitos Intestinais & $\mathbf{N}^{\circ}$ de & Frequência relativa (\%) \\
\hline Protozoários & $\mathbf{4 2 3}$ & $\mathbf{9 7 , 9 2 \%}$ \\
Endolimax nana & 178 & $41,20 \%$ \\
Blastocystis hominis & 79 & $18,3 \%$ \\
Entamoeba coli & 78 & $18,06 \%$ \\
Entamoeba histolytica & 50 & $11,57 \%$ \\
Giárdia lamblia & 36 & $8,33 \%$ \\
Iodamoeba butschlii & 2 & $0,46 \%$ \\
Helmintos & $\mathbf{9}$ & $\mathbf{2 , 0 8 \%}$ \\
Ascaris lumbricoides & 4 & $0,92 \%$ \\
Hymenolepis nana & 3 & $0,7 \%$ \\
Enterobius vermicularis & 1 & $0,23 \%$ \\
Schistosoma mansoni & 1 & $0,23 \%$ \\
Total de parasitos & $\mathbf{4 3 2}$ & $\mathbf{1 0 0 \%}$ \\
\hline
\end{tabular}

Como demonstrado por Dias et al. (2013), na cidade de Macapá - Amapá, as enteroparasitoses encontradas dentre os protozoários podemos foram Endolimax nana, Entamoeba coli, Entamoeba histolyticadispar, Giardia intestinalis, Iodamoeba bustchlii e Blastocystis hominis. Dentre as espécies de Helmintos, Ascaris lumbricoides, Trichuris trichiura, Enterobius vermiculares, Ancylostoma duodenale e Strongiloides stercoralis (Dias et al., 2013). 
Já em Santos (2020), referentes ao município de Oriximiná-PA, os parasitos encontrados foram, protozoários - Entamoeba coli, Entamoeba histolytica/Entamoeba dispar, Urbanorum spp., Blastocystis sp. e Giardia duodenalis, helmintos - Ascaris lumbricoides, Trichuris trichiura, Ancilostomídeos, Strongyloides stercoralis e Hymenolepis nana (Santos, 2020), sendo esses parasitos similares aos encontrados nesta pesquisa.

Mesmo com a singela divergência apresentada na incidência de parasitose em Marques et al. (2021) os parasitas presentes no estudo foram Helmintos: Trichuris trichiura, Ascaris lumbricoides, Ancylostoma spp., Entrobius vermicularis e Strongyloides sterco, Protozoários: Entamoeba histolytica/E. dispar, Giárdia lamblia, Blastocystis hominis, Endolimax nana e Entamoeba coli (Marques et al., 2021).

Com o conhecimento dos parasitos presentes no estudos, foi possível estabelecer as associações parasitárias recorrentes: E. nana, E. coli e Blastocystis hominis, sendo as associações observadas: E. nana e Blastocystis; E. nana e E. coli; E. nana e Giárdia; E. nana e E. histolytica; E. coli e E. histolytica; E. coli e Giárdia; E. coli e Blastocystis; E. nana e Ascaris lumbricoides; E. histolytica e Ascaris lumbricoides; Enterobius vermicularis e Giárdia; E. histolytica e Giárdia; Hymenolepis nana, E. nana e Blastocystis; E. nana, E. coli e Giárdia; E. nana, Giárdia e Blastocystis; E. nana, E. histolytica e Blastocystis; E. nana, E. coli e Blastocystis; E. nana, E. coli e E. histolytica; Hymenolepis nana, E. coli e Ascaris lumbricoides.

No gráfico 4, é possível constatar que a maior parte dos pacientes parasitados apresentaram 2 ou mais parasitas, chegando a apresentarem até 4 enteroparasitas em uma mesma amostra. Registrando-se a contagem de 94 pacientes com apenas um parasita, 146 com dois, 29 com três parasitos e 3 com quatro parasitas.

\section{GRAU DE PARASITISMO}

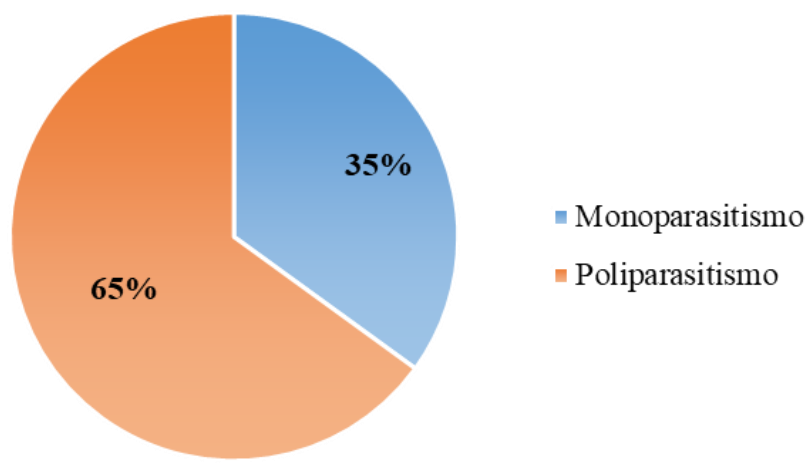

Gráfico 4. Percentual de pacientes parasitados por um ou mais parasitas - helmintos ou protozoários.

Os resultados expostos no Gráfico 4 apresentam a ocorrência de poliparasitismo mais da metade dos pacientes estudados (65\%), esse valor sendo referente a todas as idades e gêneros parasitados por dois ou mais parasitos. Tal resultado diverge as amostras analisadas no estudo realizado por Santos et al. (2020), onde $79,6 \%$ das amostras analisadas apontaram para o monoparasitismo, enquanto que em $20,4 \%$ foi encontrado poliparasitismo.

\section{Considerações finais}

A pesquisa mostrou grande relevância, pois além de ser base para indagações vindouras, expôs que as enteroparasitoses têm efeitos negativos tanto para as crianças quanto para as idades consideradas adultas, com ênfase no desenvolvimento corporal. Diante do que foi exposto, observou-se que como relatado em pesquisas relacionadas ao tema e ao objetivo da pesquisa, é comprovado a alta taxa de parasitoses no território paraense, sendo necessário colocar em prática no dia a dia as medidas profiláticas necessárias para prevenção e proteção, com ênfase nos progressos em relação a estrutura de saneamento básico e água potável.

Constatou-se que o número de crianças parasitadas foi superior ao de adolescentes/adultos, sendo prevalente em crianças e adultos o parasita comensal Endolimax nana sempre concomitantes com outras espécies, como por exemplo Blastocystis hominis e Entamoeba histolytica.

Por fim, o levantamento epidemiológico, realizado com base no banco de dados do laboratório de apoio e com pesquisas relacionadas ao tema, viabilizou um comparativo com dados da região do Pará. Contudo, é 
notável a necessidade de se realizarem outros estudos epidemiológicos sobre essas parasitoses, a fim de que se contribua para maior conscientização sobre os altos índices de incidência das mesmas no dia a dia.

\section{Referências}

Araujo Filho, H. B., Carmo-Rodrigues, M. S., Mello, C. S., Melli, L. C. F., Tahan, S., \& Morais, M. B. D. (2011). Parasitosis intestinales se asocian con menores índices de peso y estatura en niños de bajo nivel económico. Revista Paulista de Pediatria, 29(4), 521-528.

Bacelar, P. A. A., Santos, J. P., Monteiro, K. J. L., Calegar, D. A., Nascimento, E. F., \& Costa, F. A. C. 2018. Parasitoses intestinais e fatores associados no estado do Piauí: uma visão integrativa. REAS - Revista Eletrônica Acervo Saúde, 10(4), 1802-1809.

Barbosa, V. A., \& Vieira, F. O. 2013. Educação sanitária como prática de prevenção de parasitoses intestinais em creches. Acervo da Iniciação Científica, 1, 1-12.

Camello, J. T., Cavagnolli, N. I., Dalla Santa Spada, P. K. W., Poeta, J., \& Rodrigues, A. D. 2016. Prevalência de parasitoses intestinais e condições de saneamento básico das moradias em escolares da zona urbana de Caxias do Sul, Rio Grande do Sul. Scientia Medica, 26(1), ID21716.

IBGE. Instituto Brasileiro de Geografia e Estatística. 2021. Cidades: Santarém. Disponível em: https://cidades.ibge.gov.br/brasil/pa/santarem/panorama. Acesso em: 23 de nov. 2021.

Curado, A. 2020. Climas do Brasil: Tipos de climas de cada região brasileira. Conhecimento Científico. Disponível em: https://conhecimentocientifico.com/climas-do-brasil-conheca-o-clima-tipica-de-cadaregiao-brasileira/. Acesso em: 16 de agosto de 2021.

Damasceno, N. S., \& Costa, T. L. 2016. Incidência de enteroparasitoses em pacientes atendidos por um hospital universitário da cidade de Goiânia, GO, Brasil. Revista RBAC.

Deniur, J. B., Lenz, P., \& Júnior, E. R. A. Perfil clínico laboratorial de parasitoses intestinais em crianças e adolescentes em idade escolar da baixada cuiabana. Trabalho de Conclusão de Curso (Bacharelado em Biomedicina), Univag - Centro Universitário, Cuiabá, 2018.

Dias, D. S., Menezes, R. A. O., Souza, J. C., Barbosa, F. H. F., Andrade, R. F., \& Souto, R. N. P. 2013. Fatores de riscos que contribuem para as parasitoses intestinais em crianças de 0 a 5 anos em Macapá - Amapá, Brasil. Ciência Equatorial, 3(1), 17-28.

Ferreira, L. F., Chieffi, P. P., \& Araujo, A. 2012. Parasitismo não é doença. Norte Ciência, 3(1), 200-221.

Frei, F., Juncansen, C., \& Ribeiro-Paes, J. T. 2008. Levantamento epidemiológico das parasitoses intestinais: viés analítico decorrente do tratamento profilático. Cadernos de Saúde Pública, 24(12), 2919-2925.

Marques, J. R. A., Nunes-Gutjahr, A. L., \& Braga, C. E. S. 2021. Prevalência de parasitoses intestinais em crianças e pré-adolescentes no município de Breves, Pará, Brasil. Saúde e Pesquisa, 14(3), e8678.

Nascimento, N. F. 2016. Prevalência de parasitos em escolares de 0 a 15 anos em diferentes regiões do Brasil. Trabalho de Conclusão de Curso (Graduação em Ciências Biológicas) - Universidade Federal de Uberlândia, Uberlândia.

Santos, C. R. 2020. Parasitoses intestinais em amostras fecais encaminhadas ao laboratório municipal de análises clínicas de Oriximiná, Pará. Trabalho de Conclusão de Curso (Graduação em Biomedicina). Niterói, RJ: Universidade Federal Fluminese.

Silva, A. A., Silva, P. V. R., \& Rocha, T. J. M. 2018. Parasitos intestinais: frequência e aspectos epidemiológicos em usuários de um laboratório particular. Diversitas Journal, 3(2), 245-256.

Silva, L. B. M. 2017. Uma abordagem sobre as principais parasitoses intestinais mais prevalentes na infância e suas causas. Centro Universitário São Lucas, Porto Velho.

Sousa, A. C. P., Costa, L. N. G., \& Vieira, J. M. S. 2018. Prevalência de enteroparasitas em indivíduos atendidos no Laboratório Municipal de Buriti dos Lopes, Piauí, Brasil. Revista RBAC.

Vasconcelos, I. A. B., Oliveira, J. W., Cabral, F. R. F., Coutinho, H. D. M., \& Menezes, I. R. A. 2011. Prevalência de parasitoses intestinais entre crianças de 4-12 anos no Crato, Estado do Ceará: um problema recorrente de saúde pública. Acta Scientiarum. Health Sciences, 33(1), 35-41. 


\section{Minicurrículo}

Daniele Catrine de Sousa Cunha Lima. Graduada em Biomedicina. Pós-graduanda em citologia oncótica. Trabalho de Conclusão de Curso. Centro universitário da Amazônia - UNAMA, Santarém/PA.

Patrícia Spinola da Rocha. Graduação em Ciências Biológicas. Mestrado em Genética e Biologia Molecular.

Como citar: Lima, D.C.S.C, \& Rocha, P.S. 2022. Estudo da prevalência de enteroparasitoses em pacientes de um laboratório privado do município de Santarém. Pubsaúde, 8, a299. DOI: https://dx.doi.org/10.31533/pubsaude8.a299

Recebido: 29 nov. 2021.

Revisado e aceito: 4 fev. 2022.

Conflito de interesse: a autora declara, em relação aos produtos e companhias descritos nesse artigo, não ter interesses associativos, comerciais, de propriedade ou financeiros que representem conflito de interesse.

Licenciamento: Este artigo é publicado na modalidade Acesso Aberto sob a licença Creative Commons Atribuição 4.0 (CC-BY 4.0). 